CORRECTION

\title{
Author Correction: Magnesium isoglycyrrhizinate ameliorates high fructose-induced liver fibrosis in rat by increasing miR- $375-3 p$ to suppress JAK2/STAT3 pathway and TGF- $\beta 1 /$ Smad signaling
}

Yan-zi Yang ${ }^{1}$, Xiao-juan Zhao ${ }^{1}$, Hong-jiang $\mathrm{Xu}^{2}$, Shan-chun Wang ${ }^{2}$, Ying Pan ${ }^{1}$, Shui-juan Wang ${ }^{1}, \mathrm{Qiang} \mathrm{Xu}^{1}$, Rui-qing Jiao ${ }^{1}$, Hong-mei $\mathrm{Gu}^{2}$ and Ling-dong Kong ${ }^{1}$

Acta Pharmacologica Sinica (2022) 43:1616; https://doi.org/10.1038/s41401-021-00771-9

Correction to: Acta Pharmacologica Sinica https://doi.org/10.1038/ s41401-018-0194-4; published online 19 December 2018.

After publication, the authors realized the representative image of Sirius red-stained paraffin-embedded sections of liver tissue
(Fig. 1e) was misplaced. The correct figure is reproduced and presented. The conclusions are not affected by this correction. We sincerely apologize for our mistakes and any inconvenience this might have caused.

e

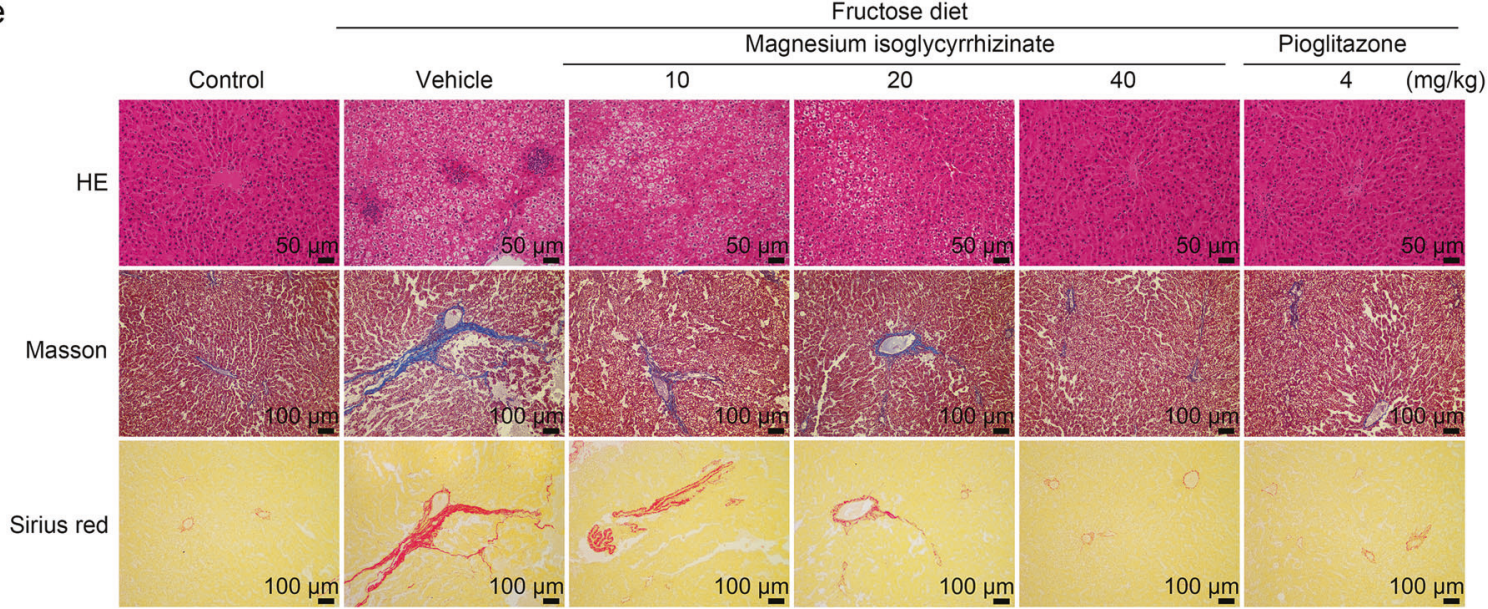

Fig. 1 Magnesium isoglycyrrhizinate alleviates liver fibrosis in high-fructose-fed rats. e Representative images of H\&E-stained (scale bar, $50 \mu \mathrm{m})$, Masson-stained (scale bar, $100 \mu \mathrm{m}$ ) and Sirius red-stained (scale bar, $100 \mu \mathrm{m}$ ) paraffin-embedded sections of liver tissues are shown.

${ }^{1}$ State Key Laboratory of Pharmaceutical Biotechnology, School of Life Sciences, Nanjing University, Nanjing 210023, China and ${ }^{2}$ Jiangsu Key Laboratory of Targeted Antiviral Research, Chia Tai Tianqing Pharmaceutical Group Co., Ltd, Nanjing 210023, China

Correspondence: Hong-mei Gu (ghm@cttq.com) or Ling-dong Kong (kongld@nju.edu.cn)

These authors contributed equally: Yan-zi Yang, Xiao-juan Zhao

The original article can be found online at https://doi.org/10.1038/s41401-018-0194-4.

Published online: 7 September 2021 